PROCEEDINGS OF THE

AMERICAN MATHEMATICAL SOCIETY

Volume 130, Number 3, Pages 855-861

S 0002-9939(01)06088-9

Article electronically published on June 8, 2001

\title{
THE SUMSET PHENOMENON
}

\author{
RENLING JIN
}

(Communicated by Carl G. Jockusch, Jr.)

\begin{abstract}
Answering a problem posed by Keisler and Leth, we prove a theorem in non-standard analysis to reveal a phenomenon about sumsets, which says that if two sets $A$ and $B$ are large in terms of "measure", then the sum $A+B$ is not small in terms of "order-topology". The theorem has several corollaries about sumset phenomenon in the standard world; these are described in sections $2-4$. One of these is a new result in additive number theory; it says that if two sets $A$ and $B$ of non-negative integers have positive upper or upper Banach density, then $A+B$ is piecewise syndetic.
\end{abstract}

\section{A THEOREM IN NON-STANDARD ANALYSIS}

Let ${ }^{*} V$ be a non-standard extension of a standard universe $V$, which contains all standard real numbers. The reader may consult [7] or [3] for basic knowledge of non-standard analysis. We denote by $\mathbf{N}$ the set of all standard non-negative integers, and denote by ${ }^{*} \mathbf{N}$ the set of all non-negative integers in ${ }^{*} V$. All integers in ${ }^{*} \mathbf{N} \backslash \mathbf{N}$ are called hyperfinite integers. For any two sets $A$ and $B$, and a binary operator $\circ$ we write $A \circ B$ for the set $\{a \circ b: a \in A$ and $b \in B\}$. An infinite initial segment $U$ of ${ }^{*} \mathbf{N}$ is called a cut if $U+U \subseteq U$. A cut is an external set (except $U={ }^{*} \mathbf{N}$ ) because $U$ cannot have a largest element. If $U$ is a cut and $c$ is a real number in ${ }^{*} V$, we write $c>U$ if $c$ is greater than every element in $U$. Given a hyperfinite integer $H$, we always write $\mathcal{H}$ for the set $\{0,1, \ldots, H-1\}$. If $U$ is a cut and $U \subseteq \mathcal{H}$, then $U$ is a "small" subset of $\mathcal{H}$ because $U$ is closed under addition, hence for every $n \in \mathbf{N}, U<H / n$. Suppose $U \subseteq \mathcal{H}$ is a cut. A set $A \subseteq \mathcal{H}$ is called $U$-nowhere dense if for any interval $[a, b] \subseteq \mathcal{H}$ of integers such that $b-a>U$, there is a subinterval $[c, d] \subseteq[a, b] \backslash A$ such that $d-c>U$. $U$-topology and $U$-nowhere dense are introduced in [6]. A real number $R$ in ${ }^{*} V$ is finite if $|R|<n$ for some standard integer $n$. Each finite $R$ has a standard part, denoted by $\operatorname{st}(R)$, and defined to be the unique standard real number $r$ such that $R$ is infinitesimally close to $r$. For any internal set $A$ we write $|A|$ for the internal cardinality of $A$ and *P $(A)$ for the set of all internal subsets of $A$. When $a$ is a standard or non-standard real number, we write $|a|$ for the absolute value of $a$.

Received by the editors September 14, 1999 and, in revised form, August 9, 2000.

2000 Mathematics Subject Classification. Primary 03H05, 03H15; Secondary 11B05, 11B13, $28 \mathrm{E} 05$.

This research was supported in part by a Ralph E. Powe Junior Faculty Enhancement Award from Oak Ridge Association Universities, a Faculty Research and Development Summer Grant from College of Charleston, and NSF grant DMS-\#0070407.

(C)2001 American Mathematical Society 
Theorem 1. Let $H$ be any hyperfinite integer and $U \subseteq \mathcal{H}$ be any cut. If $A, B \subseteq \mathcal{H}$ are two internal sets such that $\operatorname{st}(|A| / H)>0$ and st $(|B| / H)>0$, then $A \oplus_{H} B$ is not $U$-nowhere dense, where $\oplus_{H}$ is the usual addition on ${ }^{*} \mathbf{N}$ modulo $H$.

Proof. Suppose the theorem is not true. Let $U$ be a cut and $H>U$ a hyperfinite integer for which there exists a counterexample $A, B$; we will choose internal sets $A, B$ which give an "optimal" counterexample in a certain sense. Let $\alpha$ be the standard real number defined by

$$
\begin{gathered}
\alpha=\sup \left\{s t(|A| / H): H \in{ }^{*} \mathbf{N} \backslash U, A \in{ }^{*} \mathcal{P}(\mathcal{H})\right. \text { and } \\
\left.A \oplus_{H} B \text { is } U \text {-nowhere dense for some } B \in{ }^{*} \mathcal{P}(\mathcal{H}) \text { with } \operatorname{st}(|B| / H)>0\right\} .
\end{gathered}
$$

Choose a standard positive real number $\epsilon$ small enough (it will be clear later how small $\epsilon$ should be) and let $\beta$ be the standard real number such that

$$
\beta=\sup \left\{s t(|B| / H): H \in{ }^{*} \mathbf{N} \backslash U, B \in{ }^{*} \mathcal{P}(\mathcal{H})\right. \text { and }
$$

$A \oplus_{H} B$ is $U$-nowhere dense for some $A \in{ }^{*} \mathcal{P}(\mathcal{H})$ with $\left.\operatorname{st}(|A| / H)>\alpha-\epsilon\right\}$.

It is not hard to see that (1) $\beta \leqslant \alpha$, (2) $\beta>0$ and (3) $\beta<\frac{1}{2}+\epsilon$. (3) is true because otherwise there are $H, A$ and $B$ such that $A \oplus_{H} B=\mathcal{H}$ while $A \oplus_{H} B$ has to be $U-$ nowhere dense. Choose another standard positive real number $\delta$ small enough and fix $H \in{ }^{*} \mathbf{N} \backslash U$ and $A, B \in{ }^{*} \mathcal{P}(\mathcal{H})$ such that $s t(|A| / H)>\alpha-\epsilon$, st $(|B| / H)>\beta-\delta$ and $A \oplus_{H} B$ is $U$-nowhere dense. For every $k \in U$ it is easy to see by definition that

$$
\left(A \oplus_{H} B\right) \oplus_{H}[0, k]=A \oplus_{H}\left(B \oplus_{H}[0, k]\right)
$$

is also $U$-nowhere dense. Hence one has

$$
s t\left(\left|B \oplus_{H}[0, k]\right| / H\right) \leqslant \beta .
$$

By the fact that $U$ is external and the set of all $k \in{ }^{*} \mathbf{N}$ with $\left|B \oplus_{H}[0, k]\right| / H \leqslant \beta+\delta / 2$ is internal, there exists a $K \in{ }^{*} \mathbf{N} \backslash U$ such that

$$
\left|B \oplus_{H}[0,2 K]\right| / H<\beta+\delta .
$$

Since $U$ is closed under addition, one can choose $K$ small enough such that, say, $100 K<H$. Let $\mathcal{I}=\{[i K,(i+1) K-1]: i \leqslant H / K-1\}$, i.e. $\mathcal{H}$ is divided into subintervals of length $K$ except possibly the last subinterval outside of $\mathcal{I}$, which may have length less than or equal to $K$. With a small $K$ (relative to $H$ ), one can see that the last subinterval is negligible in the following arguments.

Case 1: At most one-third of the intervals in $\mathcal{I}$ are disjoint from $B$. Then one has

$$
\left|B \oplus_{H}[0,2 K]\right| / H \geqslant \frac{2}{3},
$$

which contradicts the fact that

$$
\left|B \oplus_{H}[0,2 K]\right| / H<\beta+\delta \leqslant \frac{1}{2}+\epsilon+\delta
$$

if one requires that $\epsilon<\frac{1}{12}$ and $\delta<\frac{1}{12}$.

Case 2: Not Case 1. Then $B$ is distributed among at most two-thirds of the intervals in $\mathcal{I}$. Hence there is an interval $I \in \mathcal{I}$ such that

$$
s t(|B \cap I| / K)>\beta+\delta
$$


when we choose $\delta<\frac{1}{5} \beta$. By a similar argument we can also choose an interval $J \in \mathcal{I}$ such that

$$
\operatorname{st}(|A \cap J| / K)>\alpha-\epsilon .
$$

Suppose $J=[a, a+K-1]$ and $I=[b, b+K-1]$. Let $A^{\prime}=(A \cap J)-a$ and $B^{\prime}=(B \cap I)-b$. Then

$$
A^{\prime} \oplus_{H} B^{\prime} \subseteq\left(A \oplus_{H} B\right) \ominus_{H}\left(a \oplus_{H} b\right)
$$

is $U$-nowhere dense. Since $A^{\prime} \oplus_{H} B^{\prime}=A^{\prime}+B^{\prime} \subseteq[0,2 K-1]$, then $A^{\prime} \oplus_{2 K} B^{\prime}$ is $U$-nowhere dense in $[0,2 K-1]$. Now $A^{\prime} \oplus_{K} B^{\prime}$ is the union of two $U$-nowhere dense subsets of $[0, K-1]$. Hence $A^{\prime} \oplus_{K} B^{\prime}$ is also $U$-nowhere dense in [0, $\left.K-1\right]$. But one has $s t\left(\left|A^{\prime}\right| / K\right)>\alpha-\epsilon$ and $s t\left(\left|B^{\prime}\right| / K\right)>\beta+\delta$, which contradict the maximality of $\beta$.

Remarks. (1) Theorem 1 yields a negative answer to Problem 9.13 in [6], which asks whether there is any cut $U$ for which there exist sets $A, B \subseteq \mathcal{H}$ of positive Loeb counting measure such that $A \oplus_{H} B$ is $U$-meager. Theorem 1 gives a negative answer to that problem because any set $A \subseteq \mathcal{H}$ with positive Loeb measure contains an internal subset $B$ with positive Loeb measure, which equals $s t(|B| / H)$. We write the theorem in the current form in order to avoid introducing Loeb measure, which requires that ${ }^{*} V$ be $\aleph_{1}$-saturated.

(2) For every standard real number $r<1$ and every cut $U \subseteq \mathcal{H}$, there is a set $A \in{ }^{*} \mathcal{P}(\mathcal{H})$ such that $s t(|A| / H)>r$ and $A$ is $U$-nowhere dense. So Theorem 1 is not trivial.

\section{A KNOWN RESUlT IN REAL ANALYSIS}

Let $\mathbf{R}$ denote the set of all standard real numbers. The following corollary is a well-known result in real analysis.

Corollary 1. If $A, B \subseteq \mathbf{R}$ have positive Lebesgue measure, then $A+B$ contains a non-empty open interval of real numbers.

Proof. Without loss of generality we assume that $A, B \subseteq\left[0, \frac{1}{2}\right)$. Choose any hyperfinite integer $H$ and let $\varphi$ be the function from $\mathcal{H}$ to the unit interval $[0,1]$ of real numbers, such that for every $x \in \mathcal{H}$

$$
\varphi(x)=\operatorname{st}(x / H) .
$$

Since $A$ and $B$ have positive Lebesgue measure, there are internal sets $C, D \in{ }^{*} \mathcal{P}(\mathcal{H})$ such that

$$
C \subseteq \varphi^{-1}(A), D \subseteq \varphi^{-1}(B), s t(|C| / H)>0 \text { and } s t(|D| / H)>0 .
$$

Let $U=H / \mathbf{N}$ be the cut defined by

$$
H / \mathbf{N}=\{x \in \mathcal{H}: \forall n \in \mathbf{N}(x<H / n)\} .
$$

Note that for any two numbers $x, y \in \mathcal{H} \varphi(x)=\varphi(y)$ iff $|x-y| \in U$. By Theorem 1 one has that $C \oplus_{H} D=C+D$ is not $U$-nowhere dense. Hence there is an interval $[c, d] \subseteq \mathcal{H}$ such that $d-c>U$ and $C+D$ has no gaps of length greater than $U$ in $[c, d]$. Let $\varphi(c)=a$ and $\varphi(d)=b$. It is now easy to see that

$$
A+B \supseteq \varphi[C]+\varphi[D]=\varphi[C+D] \supseteq(a, b),
$$

where $(a, b)$ is an open interval of real numbers. 
Remark. It is well known that for every $r<1$ there is a closed nowhere dense subset of the unit interval of $\mathbf{R}$ which has Lebesgue measure greater than $r$.

\section{Additions on a SEQUence of CyClic Groups}

Let $\mathbf{Z}_{n}=\{0,1, \ldots, n-1\}$ be the cyclic group of order $n$ with addition $\oplus_{n}$. Suppose $A \subseteq \mathbf{Z}_{2 n}$ with $|A|=n$. How can $A$ be distributed? $A$ could be very evenly distributed such as $A=\{2 i: i=0,1, \ldots, n-1\}$. In this case the largest gap of $A$ in every interval $[a, b] \subseteq \mathbf{Z}_{2 n}$ has length at most 1 . $A$ could also be very clustered together such as $A=[0, n-1]$. In this case $A$ has a large gap $[n, 2 n-1]$. But $A$ also has no gap in a large interval $[0, n-1]$. Can one distribute $A$ so that every large interval contains a large gap of $A$ ? In order to make sense of the largeness we need to consider the asymptotic version of the arguments. A sequence of intervals $\left\langle I_{n} \subseteq \mathbf{Z}_{n}: n \in \mathbf{N}\right\rangle$ is called large if $\lim _{n \rightarrow \infty}\left|I_{n}\right|=\infty$. A sequence of intervals $\left\langle I_{n} \subseteq \mathbf{Z}_{n}: n \in \mathbf{N}\right\rangle$ is called a gap of the sequence $\left\langle A_{n} \subseteq \mathbf{Z}_{n}: n \in \mathbf{N}\right\rangle$ if $I_{n} \cap A_{n}=\emptyset$ for every $n \in \mathbf{N}$. Following an idea in [6] one can easily construct a sequence of sets $\left\langle A_{n} \subseteq \mathbf{Z}_{n}: n \in \mathbf{N}\right\rangle$ for every $r<1$ such that $\left|A_{n}\right| / n>r$ for every $n \in \mathbf{N}$ and every large sequence of intervals $\left\langle I_{n} \subseteq \mathbf{Z}_{n}: n \in \mathbf{N}\right\rangle$ contains a large gap $\left\langle J_{n} \subseteq \mathbf{Z}_{n} \backslash A_{n}: n \in \mathbf{N}\right\rangle$. What about the sum of two sequences $\left\langle A_{n} \oplus_{n} B_{n} \subseteq \mathbf{Z}_{n}: n \in \mathbf{N}\right\rangle$ ? For each $n$ let $G_{n}$ be the function defined by

$$
G_{n}(A, I)=\max \{|J|: J \text { is an interval and } J \subseteq I \backslash A\}
$$

for each $A \subseteq \mathbf{Z}_{n}$ and each interval $I \subseteq \mathbf{Z}_{n}$.

Corollary 2. Suppose $A_{n}, B_{n} \subseteq \mathbf{Z}_{n}$ for every $n \in \mathbf{N}$ and $\inf \left\{\left|A_{n}\right| / n,\left|B_{n}\right| / n: n \in\right.$ $\mathbf{N}\}>0$. For every non-principal ultrafilter $\mathcal{F}$ over $\mathbf{N}$ there is a large sequence of intervals $\left\langle I_{n} \subseteq \mathbf{Z}_{n}: n \in \mathbf{N}\right\rangle$ such that for each $X \in \mathcal{F}$ there is a $k \in \mathbf{N}$ such that the set

$$
\left\{n \in X: G_{n}\left(A_{n} \oplus_{n} B_{n}, I_{n}\right)=k\right\}
$$

is infinite. Hence the large sequence of intervals $\left\langle I_{n}: n \in \mathbf{N}\right\rangle$ contains no large gaps of $\left\langle A_{n} \oplus_{n} B_{n}: n \in \mathbf{N}\right\rangle$.

Proof. Let $\mathcal{F}$ be a non-principal ultrafilter over $\mathbf{N}$. Suppose ${ }^{*} V$ is the ultrapower of $V$ modulo $\mathcal{F}$. Then ${ }^{*} \mathbf{N}=\mathbf{N}^{\mathbf{N}} / \mathcal{F}$. Let $H=[i d]$, the equivalence class in ${ }^{*} \mathbf{N}$ represented by the identity function $i d$ from $\mathbf{N}$ to $\mathbf{N}$. Let $A=\left[\left\langle A_{n}: n \in \mathbf{N}\right\rangle\right]$ and $B=\left[\left\langle B_{n}: n \in \mathbf{N}\right\rangle\right]$. Then one has $A, B \subseteq \mathbf{Z}_{H}, s t(|A| / H)>0$ and $s t(|B| / H)>0$. Let $U \subseteq \mathbf{Z}_{H}$ be the following cut:

$$
\left\{x \in{ }^{*} \mathbf{N}: x<[f] \text { for every } f \in \mathbf{N}^{\mathbf{N}} \text { such that } \lim _{n \rightarrow \infty} f(n)=\infty\right\} .
$$

By Theorem 1, $A \oplus_{H} B$ is not $U$-nowhere dense. So there is an interval $I \subseteq \mathbf{Z}_{H}$ with $|I|>U$ such that $G_{H}\left(A \oplus_{H} B, I\right) \in U$. Hence

$$
I=\left[\left\langle I_{n} \subseteq \mathbf{Z}_{n}: n \in \mathbf{N}\right\rangle\right]
$$

for some large sequence of intervals $\left\langle I_{n} \subseteq \mathbf{Z}_{n}: n \in \mathbf{N}\right\rangle$. Since

$$
G_{H}\left(A \oplus_{H} B, I\right)=\left[\left\langle G_{n}\left(A_{n} \oplus_{n} B_{n}, I_{n}\right): n \in \mathbf{N}\right\rangle\right] \in U,
$$

then for each $X \in \mathcal{F}$

$$
\lim _{n \in X, n \rightarrow \infty} G_{n}\left(A_{n} \oplus_{n} B_{n}, I_{n}\right) \neq \infty
$$


Hence there is a $k \in \mathbf{N}$ such that

$$
Y_{k}=\left\{n \in X: G_{n}\left(A_{n} \oplus_{n} B_{n}, I_{n}\right)=k\right\}
$$

is an infinite set.

Remarks. (1) The cut $U$ defined above cannot be $H / \mathbf{N}$, and can be $\mathbf{N}$ if and only if the ultrafilter $\mathcal{F}$ is a $\mathrm{P}$-point.

(2) When $\mathcal{F}$ is a $\mathrm{P}$-point one can require that the set $Y_{k}$ is in $\mathcal{F}$.

(3) Using other cuts, one can derive several variations of Corollary 2 .

\section{Consequences in AdDitive number theory}

Suppose $A \subseteq \mathbf{N}$. For any $m, n \in \mathbf{N}, m<n$, let

$$
d_{m, n}(A)=|A \cap[m, n-1]| /(n-m) .
$$

The term $d_{m, n}(A)$ is called the relative density of $A$ in $[m, n-1]$. The upper Banach density of $A$ is defined as

$$
B D(A)=\lim _{N \rightarrow \infty} \sup _{n-m=N} d_{m, n}(A) .
$$

A set $A \subseteq \mathbf{N}$ is called thick if $A$ contains arbitrarily long intervals, i.e. for every $k \in \mathbf{N}$ there is an $a \in A$ such that $[a, a+k] \subseteq A$. A set $A \subseteq \mathbf{N}$ is called piecewise syndetic if there is a $k \in \mathbf{N}$ such that $A+[0, k]$ is thick. These definitions can be found in [1] and [2].

Corollary 3. For any two sets $A, B \subseteq \mathbf{N}$, if $B D(A)>0$ and $B D(B)>0$, then $A+B$ is piecewise syndetic.

Proof. By the overspill principle, $B D(A)>0$ and $B D(B)>0$ imply that there are intervals $[x, y]$ and $[u, v]$ of hyperfinite length such that $\operatorname{st}\left(d_{x, y}\left({ }^{*} A\right)\right)>0$ and $s t\left(d_{u, v}\left({ }^{*} B\right)\right)>0$. By pruning both $A$ and $B$, one can assume that $y-x=v-u$. Let

$$
C=\left({ }^{*} A \cap[x, y-1]\right)-x \text { and } D=\left({ }^{*} B \cap[u, v-1]\right)-u .
$$

Let $H=2(y-x)$. Then $C, D \subseteq[0, H / 2]$, st $(|C| / H)>0$ and $s t(|D| / H)>0$. By Theorem 11 $C+D=C \oplus_{H} D$ is not $U$-nowhere dense, where $U=\mathbf{N}$. Hence there is an interval $I=[a, b]$ of hyperfinite length such that $C+D$ has no gaps of hyperfinite length in $I$. Since $C+D$ is internal, then $C+D$ has no gaps of length $>k$ for some $k \in \mathbf{N}$. So

$$
C+D+x+u=(C+x)+(D+u)={ }^{*} A \cap[x, y-1]+{ }^{*} B \cap[u, v-1]
$$

has no gaps of length $>k$ in the interval $I+x+u=J$. So ${ }^{*}(A+B)={ }^{*} A+{ }^{*} B$ has no gaps of length $>k$ in $J$. By the transfer principle, $A+B$ is piecewise syndetic.

Remarks. (1) For each $r<1$ one can find a set $A \subseteq \mathbf{N}$ such that $B D(A)>r$ and $A$ is not piecewise syndetic.

(2) Corollary 3 is a complementary result to a result in [2] and in [1] which says that if $A$ has positive upper Banach density, then $(A-A) \cap \mathbf{N}$ is syndetic. A set $A \subseteq \mathbf{N}$ is syndetic if $\mathbf{N} \backslash(A+[0, k])$ is finite for some $k \in \mathbf{N}$.

(3) Obviously the conditions $B D(A)>0$ and $B D(B)>0$ can be replaced by $\bar{d}(A)>0$ and $\bar{d}(B)>0$ because $\bar{d}(C) \leqslant B D(C)$ for every $C \subseteq \mathbf{N}$, where $\bar{d}(C)=$ $\lim \sup _{n \rightarrow \infty} d_{0, n}(C)$ is called the upper density of $C$ for $C \subseteq \mathbf{N}$. 
(4) Steven Leth pointed out to me that Corollary 3 is a consequence of Theorem 1.

Corollary 3 itself has two interesting consequences. The first consequence is related to Shnirel'man's theorem (cf. [8), which says that for any $A \subseteq \mathbf{N}$, if $\underline{d}(A)>0$ and $0,1 \in A$, then $k A=\left\{a_{1}+a_{2}+\cdots+a_{k}: a_{1}, a_{2}, \ldots, a_{k} \in A\right\}=\mathbf{N}$ for some $k \in \mathbf{N}$. In Shnirel'man's theorem the condition $\underline{d}(A)>0$ may not be replaced by $\bar{d}(A)>0$. It is easy to find an $A$ such that $\bar{d}(A)>0,0,1 \in A$, and for all $k \in \mathbf{N}, \underline{d}(k A)=0$. In the next corollary we show what we can get if we replace the condition $\underline{d}(A)>0$ by $\bar{d}(A)>0$.

Corollary 4. If $A \subseteq \mathbf{N}$ has positive upper Banach density and $a, a+1 \in A$ for some $a$, then there is a $k \in \mathbf{N}$ such that $k A$ is thick.

Proof. Left to the reader.

Remark. The condition $a, a+1 \in A$ can be replaced by $\operatorname{gcd}\left(\left(A-a_{0}\right) \cap \mathbf{N}\right)=1$, where $a_{0}$ is the least element in $A$ and $\operatorname{gcd}\left(\left(A-a_{0}\right) \cap \mathbf{N}\right)$ is the greatest common divisor of all positive integers in $A-a_{0}$.

The following result about $\mathrm{IP}$-sets gives the second interesting consequence of Corollary 3. A set of the form $F S\left(x_{n}\right)_{n=0}^{\infty}$ for some sequence $\left\{x_{n} \in \mathbf{N}: n \in \mathbf{N}\right\}$ is called an $\mathrm{IP}-$ set, where

$$
F S\left(x_{n}\right)_{n=0}^{\infty}=\left\{\sum_{n \in \alpha} x_{n}: \alpha \text { is a non-empty finite subset of } \mathbf{N}\right\}
$$

Hindman's theorem (cf. [2]) says that if an IP-set is partitioned into finitely many pieces, then one of them must contain an IP-set. By Hindman's theorem, a set of the form $t+F S\left(x_{n}\right)_{n=0}^{\infty}$ can be considered "large" because it can't be chopped into finitely many "small" pieces. But this "large-ness" is incomparable with the largeness in terms of upper density. As mentioned in [1], for each $r<1$ there is a set $A \subseteq \mathbf{N}$ with $\bar{d}(A)>r$ such that $A$ contains no subsets of the form $t+F S\left(x_{n}\right)_{n=0}^{\infty}$. In the next corollary we show what happens for $A+B$.

Corollary 5. If $A, B \subseteq \mathbf{N}$ both have positive upper Banach density, then $A+B$ contains a set of the form $t+F S\left(x_{n}\right)_{n=0}^{\infty}$.

Proof. It is easy to see that a thick set contains an IP-set. Hence by Corollary 3 there is a $k \in \mathbf{N}$ such that $A+B-[0, k]=\bigcup_{i=0}^{k}(A+B-i)$ contains an IP-set. By Hindman's theorem, there is a $t \leqslant k$ such that $A+B-t$ contains an IP-set $F S\left(x_{n}\right)_{n=0}^{\infty}$. So $A+B$ contains the set $t+F S\left(x_{n}\right)_{n=0}^{\infty}$.

\section{AdDitional REMARK}

There have been some new developments on the subject since the early version of this paper was written. I posed three questions in the early version. Now all of those three questions have been answered.

The first question asks if the following variation of Corollary 2 is true:

For any $A_{n}, B_{n} \subseteq \mathbf{Z}_{n}$ with $\inf \left\{\left|A_{n}\right| / n,\left|B_{n}\right| / n: n \in \mathbf{N}\right\}>0$, there is a sequence of intervals $\left\langle I_{n} \subseteq \mathbf{Z}_{n}: n \in \mathbf{N}\right\rangle$ and a $k \in \mathbf{N}$ such that $\lim _{n \rightarrow \infty}\left|I_{n}\right|=\infty$ and $G_{n}\left(A_{n} \oplus_{n} B_{n}, I_{n}\right)<k$. 
Recently, I found that the answer to the above question is no.

The second question asks whether one can estimate the number $k$ in Corollary[3 in terms of $B D(A)$ and $B D(B)$. I found that $k$ does not directly depend on $B D(A)$ and $B D(B)$. For any positive real numbers $\alpha$ and $\beta$ such that $\alpha+\beta<1$ and for any $k \in \mathbf{N}$, there exist two sets $A, B \subseteq \mathbf{N}$ such that $B D(A)>\alpha, B D(B)>\beta$, and $A+B+[0, k]$ is not thick.

The third question asks if one can find standard elementary proofs of Corollary 2 and Corollary 3. My original intention was that if the answers to the first and second questions should be positive, then some elementary proofs of the corollaries could yield some information on those two questions. But now we know the answers to those two questions are negative; the third question becomes less interesting. However, I did produce a standard proof of Corollary 3 in 5 .

I have also written another paper 4 on additive number theory using nonstandard analysis. In [4 I show that one can formulate and prove a theorem about upper Banach density parallel to each existing theorem about Shnirel'man density or lower density.

\section{REFERENCES}

[1] Bergelson, Vitaly, Ergodic Ramsey Theory-an Update, Ergodic theory of $\mathbf{Z}^{d}$ actions (Warwick, 1993-1994) 1-61, London Mathematical Society Lecture Note Ser. 228, Cambridge University Press, Cambridge, 1996. MR 98g:28017

[2] Furstenberg, H., Recurrence in Ergodic Theory and Combinatorial Number Theory, Princeton University Press, 1981. MR 82j:28010

[3] Henson, C. W., Foundations of nonstandard analysis-A gentle introduction to nonstandard extension, in Nonstandard Analysis: Theory and Applications, ed. by N. J. Cutland, C. W. Henson, and L. Arkeryd, Kluwer Academic Publishers, 1997. MR 99i:03085

[4] Jin, Renling, Nonstandard Methods for Upper Banach Density Problems, to appear, The Journal of Number Theory. http://math.cofc.edu/faculty/jin/research/publication.html

[5] Jin, Renling, Standardizing Nonstandard Methods for Upper Banach Density Problems, to appear, DIMACS Series, Unusual Applications of Number Theory. http://math.cofc.edu/faculty/jin/research/publication.html

[6] Keisler, H. Jerome and Leth, Steven C., Meager Sets on the Hyperfinite Time Line, The Journal of Symbolic Logic, Vol. 56 (1991), pp. 71-102. MR 93a:03074

[7] Lindstrom, T., An invitation to nonstandard analysis, in Nonstandard Analysis and Its Application, ed. by N. Cutland, Cambridge University Press, 1988. CMP 21:05

[8] Nathanson, Melvyn B., Additive Number Theory-the Classical Bases, Springer, 1996. MR 97e:11004

Department of Mathematics, College of Charleston, Charleston, South Carolina 29424

E-mail address: jinr@cofc.edu 